\title{
Versatile reduced-order model of leading-edge vortices on rotary wings
}

\author{
D. Chen, ${ }^{1,{ }^{*}}$ D. Kolomenskiy, ${ }^{2, \dagger}$ R. Onishi, ${ }^{2}$ and H. Liu ${ }^{3, \ddagger}$ \\ ${ }^{1}$ Shanghai Jiao Tong University and Chiba University International Cooperative Research Center (SJTU-CU \\ ICRC), Shanghai Jiao Tong University, 800 Dongchuan Road, Minhang District, Shanghai 200240, \\ People's Republic of China \\ ${ }^{2}$ Japan Agency for Marine-Earth Science and Technology (JAMSTEC) 3173-25, Showamachi, Kanazawa-ku, \\ Yokohama, 236-0001, Japan \\ ${ }^{3}$ Graduate School of Engineering, Chiba University 1-33, Yayoi-cho, Inage-ku, Chiba, 263-8522, Japan
}

(Received 22 February 2018; published 30 November 2018)

\begin{abstract}
The leading-edge vortex (LEV) is a universal and robust lift enhancement mechanism in biological flapping and autorotating flight. It is characterized by comparatively low Reynolds number and large angle of attack leading to separated three-dimensional flow, which has long precluded analytical approach to this problem. Here we propose a reducedorder analytical model, which is capable of delivering a fast closed-form estimate of the LEV strength and position for a revolving wing at arbitrary angle of attack. It is postulated that equilibrium state of the vortex is primarily maintained by the competing effects of vorticity production at the leading edge and its three-dimensional transport in the presence of spanwise flow and downwash. The results of the model are found consistent with the LEV strength and centroid coordinates measured previously in experiments, as well as determined from numerical solution of the Navier-Stokes equations, in a wide range of the Reynolds number. The agreement is good not only for rectangular wings, but also for bio-inspired shapes of fruit fly, bumblebee, and hawkmoth. Hence, the model offers a simple, versatile, and reliable tool for practical estimation of the LEV parameters.
\end{abstract}

DOI: 10.1103/PhysRevFluids.3.114703

\section{INTRODUCTION}

One of the key aerodynamic solutions used by small biological fliers to avoid stall, convergent among insects, bats, birds, as well as autorotating seeds, is a persistent leading-edge vortex (LEV) that remains close to the wing surface while it moves [1]. Pressure deficit in the vortex core extends to the upper surface of the wing, thus enhancing the aerodynamic forces [2]. This universal mechanism of biological flight has important potential application in the design of micro-air vehicles. Yet there has been no analytical model proposed for quick estimation of the

\footnotetext{
*Also at Graduate School of Engineering, Chiba University, 1-33, Yayoi-cho, Inage-ku, Chiba, 263-8522, Japan.

†dkolomenskiy@jamstec.go.jp; dkolom@gmail.com

${ }^{\ddagger}$ Also at Shanghai Jiao Tong University and Chiba University International Cooperative Research Center (SJTU-CU ICRC), Shanghai Jiao Tong University, 800 Dongchuan Road, Minhang District, Shanghai 200240, People's Republic of China.
}

Published by the American Physical Society under the terms of the Creative Commons Attribution 4.0 International license. Further distribution of this work must maintain attribution to the author(s) and the published article's title, journal citation, and DOI. 
LEV properties. Even though discovered almost four decades ago [3], persistent attachment of the LEV has remained a controversial subject, stimulating a growing number of physical and biological experiments, as well as numerical simulations. Literature is abundant on this topic; therefore, we refer to a dedicated review paper [4]. The growing amount of experimental and computational work during the past decade (e.g., Refs. [5-11]) opens new perspectives for reduced order modeling of the LEV of a rotating wing at large angle of attack. The latter approach has been so far less popular. We are aware of only a few prior studies that may fall in this category. Specifically, a simple formula for the axial velocity has been obtained in Ref. [12], and a more sophisticated model that involves numerical solution of an ODE for the LEV trajectory has been derived in Ref. [13].

We propose an analytical model of the LEV. It is based on the physical principles of fluid dynamics (as opposed to data-driven modeling), and it has only one free parameter (i.e., a parameter which is not constrained by the model) that is estimated using Navier-Stokes simulations. By simplifying the problem, we identify the minimum set of important hydrodynamic mechanisms and derive scaling laws. In addition to these important physical insights, the model provides approximate practical estimates of the LEV properties of a rotary wing at an arbitrarily large angle of attack. Our approach is complimentary to the existing high-precision quasisteady models based on the aerodynamic coefficients measured in experiments or numerical simulations (e.g., Ref. [14]).

It has been noticed that flapping and unilaterally rotating wings produce topologically similar LEVs. A significant number of recent studies converge to a flat rectangular plate as an archetype wing [4]. It rotates about one of its ends at a constant angle of attack, as shown in Fig. 1(a). The main geometrical and kinematic parameters are the wing tip radius $R$, the chord length $c$, the angle of attack $\alpha$, and the angular velocity $\Omega$. At startup, different experiments may have different time evolution of angular velocity, but we focus in our subsequent analysis on the steady state with constant $\Omega$, which is nominally reached during the first revolution. In addition, there may be an offset between the axis of rotation and the wing root. We consider only those cases when it is sufficiently small to be neglected (less than $0.5 c$ ). Incompressible ambient fluid is characterized by constant kinematic viscosity $v$.

A special case of this problem at $\alpha=90^{\circ}$ was scrutinized previously [15]. Vorticity production due to flow separation and its three-dimensional transport including spanwise outboard-directed drainage were found sufficient to describe the LEV equilibrium state in that case. The BrownMichael vortex model [16,17] was adapted to derive closed-form expressions for the edge vortex circulation and the position of its centroid. In Sec. II we derive similar formulas for a wing at any angle of attack $\alpha$. They are valid for any aspect ratio $R / c$ provided that the wing supports a persistent LEV. In Secs. III A and III B we test the accuracy of these formulas in a wide range of the Reynolds number $\operatorname{Re}=\Omega R c / v$ between 50 and 5350, by comparing with the data from our numerical simulations and from a laboratory experiment [7]. We then examine the model to gain further insight in the effects of spanwise flow drainage and induced downwash by varying the respective parameters in Secs. III C and III D, respectively. In Sec. IIIE the same expressions provide a reasonably accurate estimate to the LEV properties even when the wing shape is not rectangular, thus showing potential to become a versatile tool for quantifying the LEV prior to thorough measurement. Section IV contains concluding remarks.

\section{MATHEMATICAL FORMULATION OF THE MODEL}

The LEV is substituted with a steady line vortex; see Fig. 1(b). We follow the path of a selected Lagrangian element of the line vortex as its distance from the axis of rotation $r(\tau)$ increases in time $\tau$ due to the spanwise advection with velocity $V_{r}=d r / d \tau$. We define the spanwise vorticity transport coefficient $K_{s p}=V_{r} /(\Omega r)$ that can be assumed independent of $r$ [12,15]. The solution of $d r / d \tau=K_{s p} \Omega r$, up to a constant of integration $r_{0}$, is

$$
r=r_{0} \exp K_{s p} \Omega \tau
$$


(a)

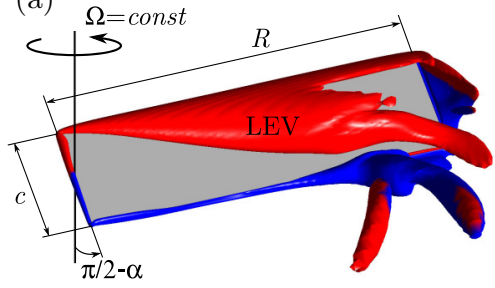

(d)

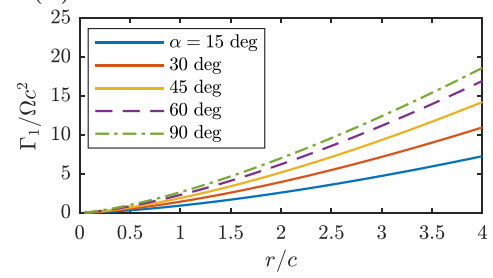

(b)

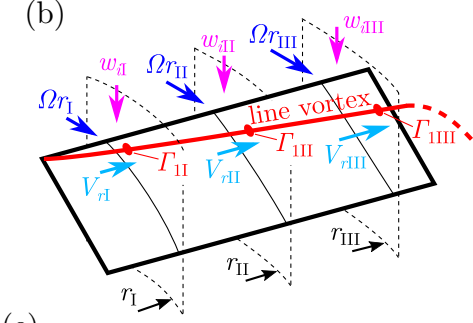

(e)

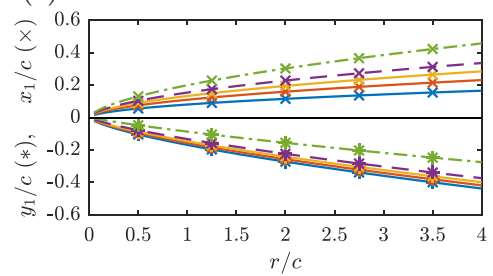

(c)
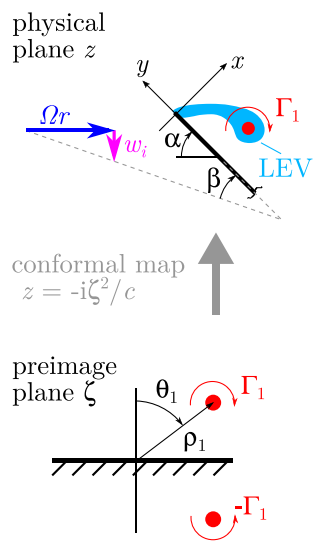

FIG. 1. (a) Flow visualization of a rectangular flat-plate wing that rotates about its root with constant angular velocity $\Omega$. $R$ is the distance from the axis of rotation to the tip edge; $c$ is the chord length. The angle of attack $\alpha$ is defined as $\pi / 2$ minus the angle between the rotation axis and the root edge. Vortices in the surrounding fluid are visualized using an isosurface of the $\lambda_{2}$ criterion, colored according of the sign of spanwise vorticity component. The red cone structure above the upper surface of the wing is the leading-edge vortex (LEV). (b) Line-vortex approximation of the LEV. A Lagrangian vortex element that originates from the leading edge of the root section is transported towards the wing tip with the spanwise velocity $V_{r}$. Simultaneously, it is swept away from the leading edge moving through the surrounding fluid with local velocity $\Omega r$. Circulation $\Gamma_{1}$ of the vortex increases with $r$ by the amount of vorticity produced at the leading edge. Spanwise variation of the LEV circulation, including its termination at the wing tip, is accompanied by chordwise vorticity, which induces downwash $w_{i}$. Subscripts "I," "II," and "III" denote the velocities sampled on three different cylindrical planes of radius $r_{\mathrm{I}}, r_{\mathrm{II}}$, and $r_{\mathrm{III}}$, respectively. (c) Two-dimensional approximation is used to describe the flow on a cylindrical plane. Spanwise evolution of the vortex is substituted with time evolution on a cylinder plane of radius that varies with time $\tau$ [Eq. (1)]. Inflow velocity $V$ is calculated as vector sum of $\Omega r$ and $w_{i}$. Circulation $\Gamma_{1}$ and position $z_{1}=x_{1}+i y_{1}$ of the vortex on a complex plane are calculated using conformal mapping between a slit plane and a half-plane. Polar coordinates $\rho_{1}$ and $\theta_{1}$ are used for describing the position of the vortex and its mirror image on the preimage plane $\zeta$, using the coordinate transform $\zeta=\rho \exp [i(\pi / 2-\theta)]$. (d) Spanwise profiles of the normalized LEV circulation (14) of a wing with aspect ratio $R / c=4$ at Reynolds number $\operatorname{Re}=\Omega R c / v=500$, evaluated at five different angles of attack $\alpha$. (e) Normalized perpendicular, $x_{1}$, and parallel, $y_{1}$, coordinates of the vortex centroid with respect to the leading edge; see (13).

We therefore reduce the steady three-dimensional vortex to an unsteady two-dimensional BrownMichael vortex on a complex plane [Fig. 1(c)], and the problem is solved using conformal mapping $z=-i \zeta^{2} / c$. The solution closely follows the procedure described in Ref. [15] for the special case $\alpha=90^{\circ}$, with the difference that it now accounts for a nonzero velocity component parallel to the wing. The inflow complex velocity is defined as $V=V_{x}+i V_{y}$, where $V_{x}=V \sin \beta$ and $V_{y}=$ $-V \cos \beta$. Its magnitude is equal to $V=\Omega r \sqrt{1+\kappa^{2}}$, where $\kappa=w_{i} / \Omega r$. Its phase angle contains a correction for the induced angle of attack such that $\beta=\alpha-\arctan \kappa$. The flow in the vicinity of the leading edge and the point vortex is described by the complex potential

$$
W=V_{x} \zeta-V_{y} \frac{\zeta^{2}}{c}-\frac{\Gamma_{1}}{2 \pi i} \ln \frac{\zeta-\zeta_{1}}{\zeta-\zeta_{1}^{*}}
$$

The Brown-Michael equation of vortex motion in the physical plane is written as

$$
\frac{d z_{1}}{d \tau}+z_{1} \frac{1}{\Gamma_{1}} \frac{d \Gamma_{1}}{d \tau}=\tilde{u},
$$


where on the right-hand side $\tilde{u}$ is the desingularized velocity. Its complex conjugate is mapped onto the preimage plane, $\tilde{v}^{*}=\tilde{u}^{*}(d z / d \zeta)$, and related to the complex potential $W$,

$$
\tilde{v}^{*}=\frac{\partial W}{\partial \zeta}-\frac{i \Gamma_{1}}{2 \pi}\left(\frac{1}{\zeta-\zeta_{1}}+\frac{1}{2 \zeta_{1}}\right)
$$

The position of the point vortex on the preimage plane $\zeta_{1}$ is thus described by the equation

$$
\begin{gathered}
\left(2 i \zeta_{1}^{*}+\frac{i \zeta_{1} \zeta_{1}^{*}}{\zeta_{1}-\zeta_{1}^{*}}\right) \frac{d \zeta_{1}^{*}}{d \tau}-\frac{i \zeta_{1}^{* 3}}{\zeta_{1}\left(\zeta_{1}-\zeta_{1}^{*}\right)} \frac{d \zeta_{1}}{d \tau}+\frac{i \zeta_{1}^{* 2}}{V_{x}} \frac{d V_{x}}{d \tau} \\
=-i V_{y} c+\frac{i c^{2}}{2 \zeta_{1}}\left[V_{x}-\frac{i \Gamma_{1}}{2 \pi\left(\zeta_{1}-\zeta_{1}^{*}\right)}-\frac{i \Gamma_{1}}{4 \pi \zeta_{1}}\right]
\end{gathered}
$$

with the initial condition $\zeta_{1}(0)=0$.

It is convenient to solve this problem in polar coordinates such that $\zeta=\rho \exp [i(\pi / 2-\theta)]$; see Fig. 1(c). The Kutta condition, satisfied if $\partial W / \partial \zeta=0$ at $\zeta=0$, relates the circulation with the position of the vortex as

$$
\Gamma_{1}=\pi V_{x} \rho_{1} / \cos \theta_{1} .
$$

Let us define variables $\Upsilon=V_{x} \rho_{1}^{3} / c^{2}, \Theta=\sin \theta_{1}$, and $\tilde{\tau}=\int_{-\infty}^{\tau} V_{x}^{2}\left(\tau^{\prime}\right) d \tau^{\prime}$. The latter integral can be evaluated using (1) as $\tilde{\tau}=0.5 \Omega r^{2}\left(1+\kappa^{2}\right) \sin ^{2} \beta / K_{s p}$, assuming constant downwash factor $\kappa$. From (5), for $\beta>0$, we derive a system of equations in real variables,

$$
\begin{aligned}
\frac{d \Upsilon}{d \tilde{\tau}} & =\frac{\Theta}{4}-\left(1-\Theta^{2}\right) \frac{\rho_{1}}{c} \cot \beta, \\
\Upsilon \frac{d \Theta}{d \tilde{\tau}} & =\frac{1-2 \Theta^{2}}{8}+\Theta\left(1-\Theta^{2}\right) \frac{\rho_{1}}{c} \cot \beta .
\end{aligned}
$$

We combine the two equations to eliminate the terms that contain $\rho_{1}$ and derive an equation for $\Upsilon \Theta, d(\Upsilon \Theta) / d \tilde{\tau}=1 / 8$, which is readily integrated using the initial condition $\Upsilon(0)=0$, as long as the vortex does not coincide with the boundary at any $\tau>0$. We obtain

$$
\Upsilon=\frac{\tilde{\tau}}{8 \Theta} \quad \text { and } \quad \rho_{1}=\frac{\tilde{\tau}^{1 / 3} c^{2 / 3}}{2\left(\Theta V_{x}\right)^{1 / 3}}
$$

making use of the definition of $\Upsilon$, and substitute these results in the second equation (7) to eliminate $\Upsilon$ and $\rho_{1}$ from the equation. This leads to an equation for $\Theta$ only,

$$
\frac{d \Theta}{d \hat{\tau}}=\frac{\Theta}{\hat{\tau}}-\frac{2 \Theta^{3}}{\hat{\tau}}+\frac{\Theta^{5 / 3}\left(1-\Theta^{2}\right)}{\hat{\tau}^{5 / 6}}, \quad \hat{\tau}=\frac{(4 \cot \beta)^{6}}{2 K_{s p} \Omega c^{2}} \tilde{\tau},
$$

and, using the definition $\Theta=\sin \theta_{1}$, to

$$
\frac{d \theta_{1}}{d \hat{\tau}}=\frac{1}{\hat{\tau}} \tan \theta_{1} \cos 2 \theta_{1}+\frac{1}{\hat{\tau}^{5 / 6}} \sin ^{5 / 3} \theta_{1} \cos \theta_{1} .
$$

An approximate solution that satisfies the initial condition $\theta_{1}(0)=\pi / 4$ and has the correct asymptotic decay as $\hat{\tau} \rightarrow \infty$ can be written as

$$
\begin{gathered}
\theta_{1}=\frac{\pi}{2} \frac{2+\pi \hat{\tau}^{1 / 12}+B \pi \hat{\tau}^{1 / 24}}{4+\pi \hat{\tau}^{1 / 12}+B \pi \hat{\tau}^{1 / 24}}+O\left(\hat{\tau}^{-1 / 6}\right), \\
\text { where } \hat{\tau}=\frac{1024 \cos ^{6} \beta}{K_{s p}^{2} \sin ^{4} \beta}\left(1+\kappa^{2}\right) \frac{r^{2}}{c^{2}},
\end{gathered}
$$

and the parameter $B$ controls the accuracy of approximation at intermediate $\hat{\tau} . B=-0.7$ is sufficient to ensure the accuracy of (11) within the error bound of 0.05 uniformly. With $\theta_{1}$ known, 
we use the second equation (8) to evaluate $\rho_{1}$, calculate the point vortex position in the preimage plane, $\zeta_{1}=\rho_{1} \exp \left[i\left(\pi / 2-\theta_{1}\right)\right]$, and use the conformal mapping to determine the position in the physical plane,

$$
z_{1}=c \frac{\left(1+\kappa^{2}\right)^{1 / 3} \sin ^{2 / 3} \beta}{\left(16 K_{s p} \sin \theta_{1}\right)^{2 / 3}}\left(\frac{r}{c}\right)^{2 / 3} \exp i\left(\frac{\pi}{2}-2 \theta_{1}\right) .
$$

The circulation evaluates from (6) as

$$
\Gamma_{1}=\Omega c^{2} \frac{\pi\left(1+\kappa^{2}\right)^{2 / 3} \sin ^{4 / 3} \beta}{\left(16 K_{s p} \sin \theta_{1}\right)^{1 / 3} \cos \theta_{1}}\left(\frac{r}{c}\right)^{4 / 3} .
$$

The vorticity transport coefficient $K_{s p}$ was introduced in Ref. [15] as a parameter that measures the efficiency of spanwise vorticity drainage, and it was related to the rate at which the spanwise flow of the fluid trapped in the recirculation bubble evacuated the LEV vorticity. Since the width of the bubble scales with the effective angle of attack $\beta$ as $c \sin \beta$, it is plausible to assume that

$$
\begin{aligned}
& K_{s p}=K_{90} \sin \beta, \\
& K_{90}=\min \left\{0.078 \sqrt{\operatorname{Re}_{1}}, 0.8\right\},
\end{aligned}
$$

where $\operatorname{Re}_{1}=\Omega c^{2} / \nu$, and $K_{90}$ is an empirical fit for $K_{s p}$ at $\beta=90^{\circ}$. The effect of $K_{s p}$ will be further discussed in Sec. III C.

The downwash factor $\kappa$ can be roughly estimated as the velocity induced by a vortex ring divided by the characteristic velocity $\Omega R$. Using the Biot-Savart law to calculate the velocity at the center of the vortex ring of radius $R$, we find $\kappa=\Gamma_{\text {tip }} /\left(2 \Omega R^{2}\right)$, where $\Gamma_{\text {tip }}$ is the tip vortex circulation that can be obtained by evaluating (14) at $r=R$, because the wing-tip vortex that forms the ring actually originates from the wing tip; see Fig. 1(b). This approach results in a non-linear algebraic equation for $\kappa$ that can be approximately solved using fixed-point iteration. It converges rapidly; therefore, in practice we perform only one iteration. First, $\Gamma_{\text {tip }}$ is estimated using (14) with $\kappa=0$. It yields a closer approximation for $\kappa$,

$$
\kappa=\frac{\pi \sin \alpha}{\left(32 K_{90}\right)^{1 / 3}}\left(\frac{R}{c}\right)^{-2 / 3} .
$$

The effect of $\kappa$ will be further discussed in Sec. III D. We conclude this section by emphasizing that formulas (11)-(17) combined constitute closed-form expressions for $z_{1}$ and $\Gamma_{1}$.

\section{DISCUSSION}

\section{A. The angle of attack and the Reynolds number effects}

Sample plots of normalized $\Gamma_{1}, x_{1}=\Re z_{1}$, and $y_{1}=\Im z_{1}$ as functions of $r / c$ and $\alpha$ are displayed in Figs. 1(d) and 1(e). The shape of the profiles $\Gamma_{1}(r)$ is mainly determined by the factor $r^{4 / 3}$ in (14), but the power-law growth is distorted by variation of $\theta_{1}$ with $r . \Gamma_{1}$ increases between $\alpha=15^{\circ}$ and $60^{\circ}$ but varies remarkably less between $60^{\circ}$ and $90^{\circ}$. The shape of $x_{1}(r)$ and $y_{1}(r)$ resembles $r^{2 / 3}$. The perpendicular component $x_{1}(r)$ increases with $\alpha$, i.e., the LEV moves further away from the wing surface when the angle of attack increases. In contrast, the parallel component $y_{1}(r)$ decreases in magnitude as $\alpha$ increases. The limiting result at $\alpha=90^{\circ}$ should be interpreted with caution because the downwash model (17) does not account for the trailing edge vortex, which is, in that particular case, equally strong as the LEV.

Computational fluid dynamics (CFD) is employed in this study to assess the accuracy of the above theoretical estimates. The incompressible Navier-Stokes equations are solved using a commercial finite-volume-based code ANSYS CFX (version 14.5). The CFD model for simulation is similar to our previous work [15]; however, in the present study we use a shorter rectangular-plate wing that has length $4 c$ that is inclined to the vertical axis as shown in Fig. 1(a). The pivot point is 

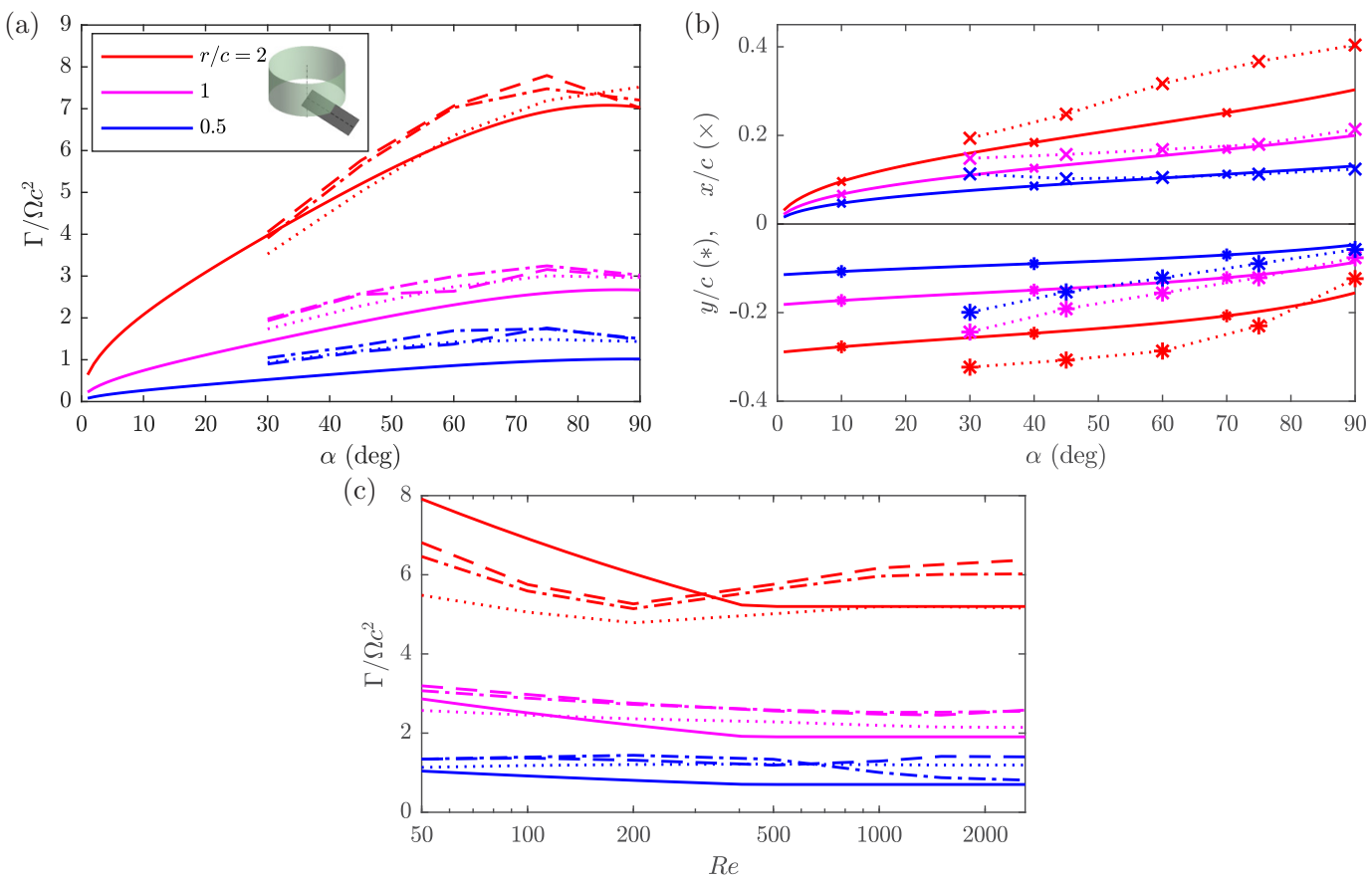

FIG. 2. Normalized values of (a) circulation $\Gamma$ and (b) perpendicular, $x$, and parallel, $y$, coordinates of the vortex with respect to the leading edge, as functions of the angle of attack $\alpha$, at a constant Reynolds number $\operatorname{Re}=\Omega R c / v=500$. (c) LEV circulation as a function of $\operatorname{Re}$, at $\alpha=45^{\circ}$. Continuous lines correspond to the theoretical estimates (14) for $\Gamma_{1}$ and (13) for $z_{1}$, evaluated at spanwise stations $r=0.5 c, c$, and $2 c$, which correspond to $12.5 \%, 25 \%$, and $50 \%$ of the wing length $(R=4 c)$, respectively. Discontinuous lines show the normalized circulation obtained from the CFD simulations. Dotted, dash-dot, and dashed lines correspond to, respectively, $\phi=84^{\circ}, 180^{\circ}$, and $270^{\circ}$ angular distance travelled by the wing. Inset shows the half-cylinder surface $r=$ const, $0 \leqslant y^{\prime} \leqslant L_{y} / 2,0 \leqslant \phi^{\prime}<2 \pi$ used as domain of integration of the radial vorticity $\omega_{r}$, which yields $\Gamma=\int_{0}^{2 \pi} \int_{0}^{L_{y} / 2} \omega_{r} r d y^{\prime} d \phi^{\prime}, x+i y=\frac{1}{\Gamma} \int_{0}^{2 \pi} \int_{0}^{L_{y} / 2}\left(r \phi^{\prime}+i y^{\prime}\right) \omega_{r} r d y^{\prime} d \phi^{\prime} . L_{y}$ is the CFD domain height.

located at the center of root chord, i.e., the offset between the axis of rotation and the wing root is equal to zero. The wing is immersed in a spherical inner domain, and both rotate around the vertical axis with angular velocity $\Omega(t)=\frac{1}{2} \Omega_{s t d}\left[1-\cos \left(\pi t / t_{a c}\right)\right]$ during the startup period $t<t_{a c}$ and with constant angular velocity $\Omega_{\text {std }}$ later. The acceleration time is equal to $t_{a c}=0.054 \times 2 \pi / \Omega_{s t d}$. Numerical validation of the CFD method can be found in part 1 of the Supplemental Material [18].

Figures 2(a) and 2(b) present a comparison between the CFD results and the corresponding theoretical estimates in a form that helps to focus on the effect of angle of attack $\alpha$. In Fig. 2(a) lines show $\Gamma(\alpha)$ for three selected spanwise locations $r=0.5 c, c$, and $2 c$, all belonging to the proximal part of the wing that supports an equilibrium-state LEV. The CFD and the theory both show the same trend of $\Gamma$ increasing with $\alpha$ until saturation at large $\alpha$. It is followed by a slight decrease between $\alpha=75^{\circ}$ and $90^{\circ}$, although this effect is marginal in the theory. Figure 2(b) shows that the LEV core moves farther away from the wing surface ( $x$ increases) as $\alpha$ increases. At the same time, the projected position of the LEV on the wing surface moves closer to the leading edge ( $|y|$ decreases). Variation of $\Gamma$ with Re, illustrated in Fig. 2(c), is comparatively weak. The theory slightly overestimates $\Gamma$ at low Re but underestimates it at large Re.

\section{B. Comparison with a laboratory experiment}

Some recent laboratory experiments using flapping or rotary rectangular flat-plate wings have focused on quantifying the LEV size and strength $[6,7,10]$. When referring to such measurements, 

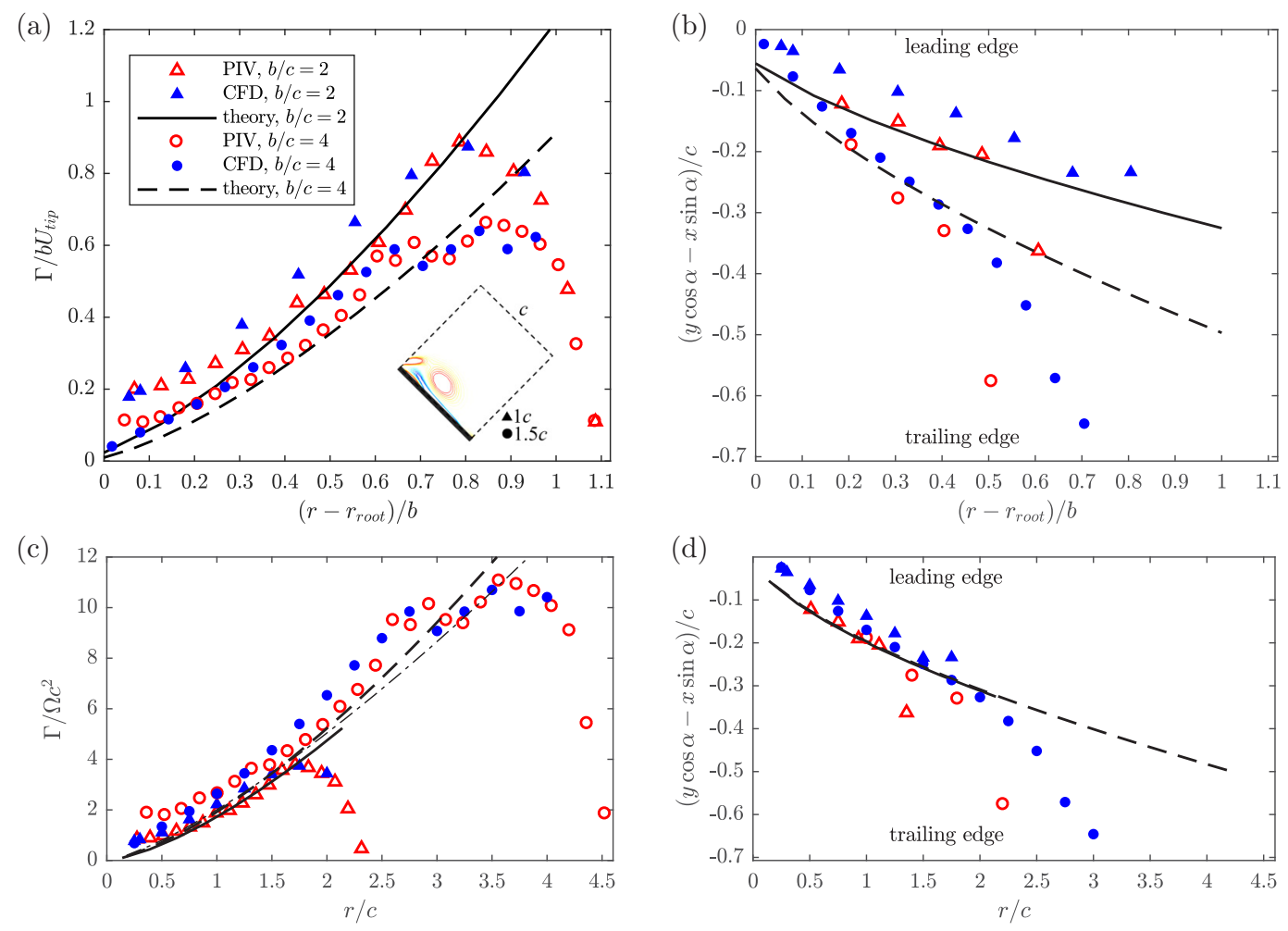

FIG. 3. Comparison between the theory (black lines), PIV measurements [7] (red markers), and CFD results (blue markers) at the rotation angle $\phi=108^{\circ}$. (a) Dimensionless LEV circulation and (b) LEV centroid position relative to the leading edge, viewed from above in the direction of the axis of rotation. Normalization is as in Ref. [7], where $b$ is the wing length from the root to the tip, $r_{\text {root }}$ is the distance from the axis of rotation to the wing root, and $U_{\text {tip }}=\Omega\left(r_{\text {root }}+b\right)$. The domain of integration for calculating $\Gamma$ is shown in the inset. (c) Circulation normalized with $\Omega c^{2}$ and (d) position relative to the leading edge, plotted as functions of the dimensionless distance from the axis of rotation $r / c$. The dash-dot line in panel (c) corresponds to $2(r / c)^{4 / 3}$. Panels (b) and (d) show the view from the top in the direction parallel to the rotation axis such that $(y \cos \alpha-x \sin \alpha) / c=0$ corresponds to the leading edge and -0.707 to the trailing edge, as required for comparison with the results of the PIV measurement shown in Ref. [7].

it is important to bear in mind the specifics of the vortex identification methods that may partly account for the variability across different results. In particular, there is no unique definition of the LEV circulation $\Gamma$. We prefer the one based on averaging [over a cylindrical surface schematically shown in the inset of Fig. 2(a)] of the radial vorticity component in cylindrical coordinates for its better compatibility with the Brown-Michael model. This is the method used in the previous section. A more popular method is to integrate the positive spanwise vorticity in a small flat rectangular window, which is more straightforward when using particle image velocimetry (PIV). For instance, it has been applied earlier [7] to evaluate the "total" circulation $\Gamma$ and LEV center trajectories for wings of aspect ratio 2 and 4 from stereoscopic digital PIV measurement. The wings were tested at $\operatorname{Re}=5350$ and $\operatorname{Re}=2600$, respectively. The angle of attack was equal to $\alpha=45^{\circ}$ for both. The results of those experiments are compared in Fig. 3 with their respective theoretical estimates, using the same normalization as in Ref. [7]. Note that the aspect ratio is defined in this case as $b / c$, where $b$ is the wing length from the root to the tip. The wing root is offset from the axis of rotation by a distance of $r_{\text {root }}=0.14 c$ for $b / c=2$ and $r_{\text {root }}=0.18 c$ for $b / c=4$. 
For the circulation [Fig. 3(a)], the agreement is good in the middle part of the wing from $(r-$ $\left.r_{\text {root }}\right) / b=0.2$ to 0.8 , where the LEV may burst but remains fully contained within the window. The nonzero $\Gamma$ at the wing root in the experiment is caused by the offset between wing root and rotation axis in the setup. This effects is stronger in the $b / c=2$ case probably because $r_{\text {root }} / b$ is larger, which means that the near-root region extends over a larger portion of the wing span. For the vortex centroid [Fig. 3(b)], the agreement is remarkably good until the midspan but rapidly degrades when approaching the wing tip. This is explained by the differences in the LEV centroid identification method: the experiment [7] considered only the primary core from the separated LEVs, but the theory points to a weighted average position between the primary and the secondary cores since both contribute to the Kutta condition and to the LEV circulation. It is therefore not surprising that the agreement near the wing tip for the vortex centroid position is less good than for the circulation. As the aspect ratio changes from 2 to 4 , the experiment and the theory both show the same trend of decreasing dimensionless circulation and increasing distance from the leading edge to the vortex core.

In addition, we have implemented CFD simulations that accurately reproduce the conditions of the experiments, including the root offset $r_{\text {root }}$. The normalized CFD circulation shown in Fig. 3(a) has been calculated from the velocity field using the same method as in the experiment, i.e., by numerical integration over a rectangle that extends over a distance of $1 c$ above the plate for $b / c=2$ and $1.5 c$ above the plate for $b / c=4$, for ease of direct comparison. The CFD points fall slightly above the experiment data between $\left(r-r_{\text {root }}\right) / b=0.3$ to 0.7 and practically overlap with the experiment data otherwise. The difference with respect to the theory is of the same order of magnitude for the CFD as for the experiment, and it represents the combined effect of all physical phenomena not taken into consideration by the theory.

The CFD and the experiment both show the same saturation of $\Gamma$ in Fig. 3(a) near the wing tip [from $\left(r-r_{\text {root }}\right) / b=0.7$ to 1 ], where the LEV detaches and escapes from the rectangular window. However, there is a large discrepancy between the respective LEV trajectories over the distal part of the wing $\left[\left(r-r_{\text {root }}\right) / b>0.5\right]$; see Fig. 3(b). The latter effect can be explained by the differences in the vortex identification procedure; see part 2 of the Supplemental Material [18] for more discussion on this topic.

An important corollary of (13) and (14) is that, once the LEV parameters are normalized using $1 / \Omega$ as the timescale and $c$ as the length scale, the LEV circulation varies nominally as $r^{4 / 3}$, and the LEV centroid position varies as $r^{2 / 3}$. This normalization makes the results corresponding to $b / c=4$ and 2 virtually collapse on the same line, as shown in Figs. 3(c) and 3(d). There only remains a minor difference due to the influence of $K_{s p}$ and $\kappa$ that take different values in these two cases. Neglecting these subtle effects, we obtain a short formula

$$
\Gamma_{45^{\circ}}=2 \Omega c^{2 / 3} r^{4 / 3}
$$

that provides a remarkably simple and accurate estimate to the LEV circulation for $\alpha=45^{\circ}$ and $0.5 c<r<R-0.5 c$. The discrepancies near the wing root and near the wing tip are due to the limitations of the theoretical model and differences in the vortex identification methods.

\section{The effect of spanwise vorticity transport}

The coefficient of spanwise vorticity transport $K_{s p}$ determines the rate of vorticity evacuation in the spanwise direction relative to the local incoming flow velocity. It depends on the Reynolds number $\operatorname{Re}=\Omega R c / v$ and the effective angle of attack $\beta$, the latter being related to the width of the recirculation bubble inside which the vorticity transport takes place. Therefore, an approximate relation $K_{s p}(\operatorname{Re}, \beta)=K_{90}(\mathrm{Re}) \sin \beta$ holds, where $K_{90}$ is the vorticity transport coefficient of a revolving flat plate oriented perpendicular to the incoming flow that has been introduced in Ref. [15] and described as a function of the root-based Reynolds number $\operatorname{Re}_{1}=\operatorname{Re} \times(c / R)=\Omega c^{2} / v$ in the range between 8 and 83 . Figure 4 displays this dependence on a semilogarithmic scale. Green circles 


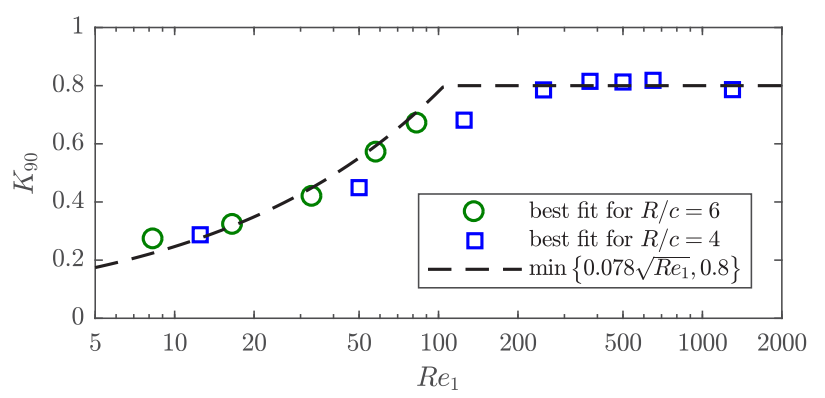

FIG. 4. Estimates of the spanwise vorticity transport coefficient $K_{90}$ as a function of the root-based Reynolds number $\operatorname{Re}_{1}=\Omega c^{2} / \nu$. The values shown with green circles best fit the theoretical estimate to the results of a CFD model with aspect ratio 6; see Ref. [15]. Blue squares depict similar results for a wing of aspect ratio 4, in a wider range of $\mathrm{Re}_{1}$. The dashed line corresponds to the empirical formula (16).

show the values of $K_{90}$ that best fit the theoretical estimate to the CFD data of a wing with $R / c=6$,

$$
\sqrt{\left(\frac{\Gamma^{\text {Theory }}-\Gamma^{\mathrm{CFD}}}{\Gamma^{\mathrm{CFD}}}\right)^{2}+\left(\frac{\left|z^{\text {Theory }}-z^{\mathrm{CFD}}\right|}{\left|z^{\mathrm{CFD}}\right|}\right)^{2}} \rightarrow \min .
$$

In the present study, we are interested in a wider range of the Reynolds number. We have therefore added points that best fit the CFD data of a wing with $R / c=4$. In the interval of $\operatorname{Re}_{1}$ below 100 $(\operatorname{Re}<400)$, these points follow the already known square-root trend. However, when the Reynolds number becomes larger, we find that $K_{90}$ saturates at the level of about 0.8 . Since $K_{90}$ is an integral quantity related to the momentum transport in a flow with fixed separation points, such Reynolds number invariance is not surprising and is expected to hold true for all larger Re. We therefore fit the low- and the high-Re regions separately and obtain the empirical relation (16) that we use further.

Let us proceed with a more practical case of $\alpha=45^{\circ}$. The aspect ratio is set to $R / c=10$ in order to mitigate the wing-tip effect and ensure the results be relevant to any sufficiently long wing. We focus on the effect of the spanwise vorticity transport coefficient $K_{s p} \approx 0.55 K_{90}$ in this case (if $\alpha$ and $R / c$ are fixed, $\sin \beta$ is approximately constant because the Reynolds number effect on $\beta$ is small). Smaller $K_{s p}$ implies less efficient spanwise transport and, as a consequence, faster approach of the LEV core to the trailing edge. Figure 5(a) shows a view perpendicular to the wing and three sample LEV centroid tracks. The yellow line corresponds to the largest possible $K_{90}=$ 0.8 , according to (16). Equivalently, it corresponds to $\mathrm{Re}_{1}=105$ or greater. It intersects the line $y / c=0.5$ at $r_{\text {crit }} / c \approx 6$. As $\operatorname{Re}_{1}$ and $K_{90}$ decrease, the intersection point $r_{\text {crit }}$ shifts towards the wing root. A parameter sweep in $K_{90}$ reveals linear dependence of $r_{\text {crit }}$ on this quantity; see Fig. 5(b). However, the realizable values of $r_{\text {crit }}$ are in the interval from 0 to $6 c$. This quantity is important for its relevance to the LEV stability. Interaction with the trailing edge being one of the factors that limits the LEV growth and provokes its detachment [19], $y / c=0.5$ can be taken as the rearmost position of the center of a steady LEV. It follows that no wing can support an attached LEV beyond $r / c=6$, or, which is equivalent, the wing should be shorter than $R / c=6$ to fully benefit from an attached LEV. In addition, we observe a trend for the maximum span of the attached LEV to shrink when $\operatorname{Re}_{1}$ is decreased.

It is worth noting that the effect of rotational accelerations such as the centripetal acceleration and the spanwise component of the Coriolis acceleration are implicitly included in $K_{s p}$. Hence, parameter sweep in $K_{90}$ is equivalent to numerical manipulation of these rotational acceleration components, while (16) fixes the value $K_{90}$ that corresponds to the experimentally realizable flow at a given $\mathrm{Re}_{1}$. Also note that our estimate of $r_{\text {crit }} / c=6$ is larger than the value of 4 determined from PIV flow visualization [20]. The model is asymptotically accurate when the LEV is near the leading 

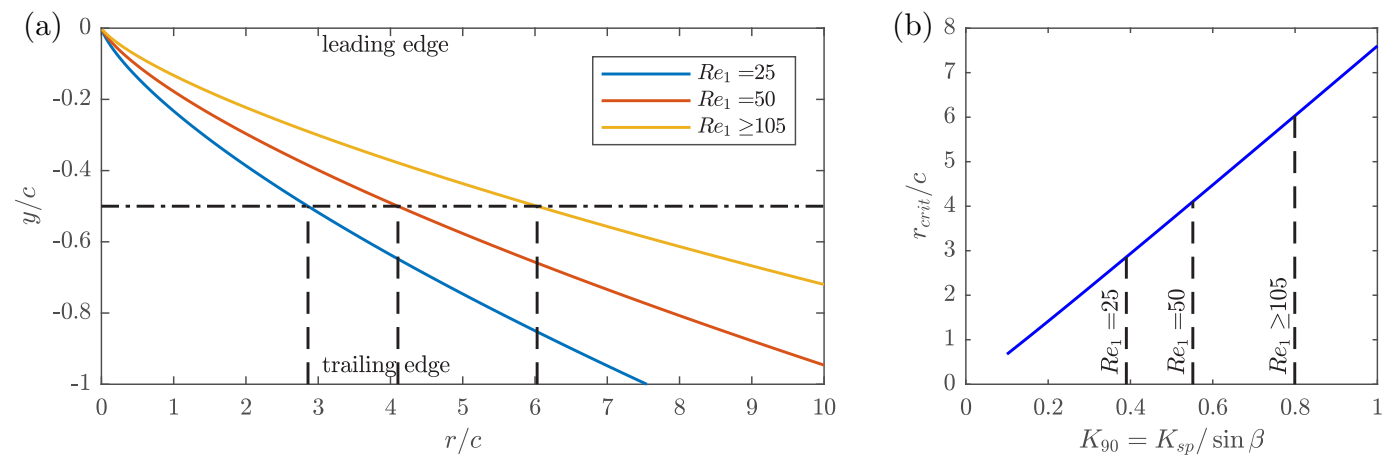

FIG. 5. (a) Chordwise component of the LEV centroid position versus the spanwise distance from the axis of rotation, for three different values of the root-based Reynolds number $\mathrm{Re}_{1}$. Intersection with the dash-dot line plotted at $y / c=0.5$ determines the critical spanwise distance $r_{\text {crit }}$. All distances are normalized with $c$. The view is in the direction perpendicular to the wing surface such that $y / c=0$ corresponds to the leading edge and -1 to the trailing edge. (b) $r_{\text {crit }}$ plotted as a function of the spanwise vorticity transport parameter $K_{90}$, the latter being varied in a hypothetical range from 0.1 to 1 . The wing has the aspect ratio $R / c=10$, and it operates at $\alpha=45^{\circ}$.

edge and far from the trailing edge. Near the detachment point, the influence of the trailing edge and variation in the spanwise velocity should be taken into account for a more accurate description of the LEV track and improved prediction of $r_{\text {crit }}$.

\section{The effect of downwash}

The downwash formula (17) has been derived by approximating the wake with a vortex ring. This wake model is arguably the simplest and at the same time reasonable while the first revolution is taking its course. When the second revolution begins, the wing encounters its own wing tip and starting vortices generated in the beginning of the motion, which implies that a more complex wake model may become necessary. A spiral vortex model is likely to be suitable for the fully developed wake. However, to avoid entering technicalities in this section, let us adhere to the same functional relation (17) as obtained earlier, and illustrate the effect of downwash by comparing between three hypothetic situations: zero $(\kappa=0)$, moderate $[\kappa$ as given by $(17)]$, and strong downwash $[\kappa$ twice as large as in (17)]. Note that, by this definition, the moderate downwash corresponds to the first revolution of the wing, but the strong downwash has no intended interpretation in terms of flight condition: it is stronger than the induced velocity in continuous rotation, and it may be equivalent to axial climb with some suitable velocity.

In all cases, $\Gamma$ starts from zero at $\alpha=0$ and rapidly increases with $\alpha$ when it is small, as illustrated in Fig. 6(a) for $\operatorname{Re}=500, R / c=4, r / c=2$. The fastest growth corresponds to the downwash intensity $\kappa=0$, in which case the maximum circulation is attained at $\alpha=60^{\circ}$, followed by a moderate decrease of $\Gamma(\alpha)$. An interesting special case is $\alpha=90^{\circ}$, such that $\theta_{1}=\pi / 4$ and the formulas (13)-(14) reduce to their simpler analogues derived in Ref. [15]. However, when $\alpha$ is slightly less that $90^{\circ}, \Gamma$ drops by a small amount. This effect can be attributed to the truncation error in (11). The vortex centroid position is the most sensitive to $\alpha$ when $\kappa=0$; see Fig. 6(b). In particular, the chordwise position $y$ varies from a finite negative value at $\alpha=0$ to zero at $\alpha=90^{\circ}$, with some slight overshoot due to the approximation error mentioned earlier.

When downwash is activated, $\Gamma$ decreases with $\kappa$ if $\alpha<60^{\circ}$, and increases otherwise. The peak shifts to larger $\alpha$ and eventually disappears. The chordwise position $y$ of the vortex becomes practically independent of $\alpha$ when the downwash becomes strong. On the other hand, the position of the vortex $x$ perpendicular to the wing is not so sensitive to the downwash intensity. 

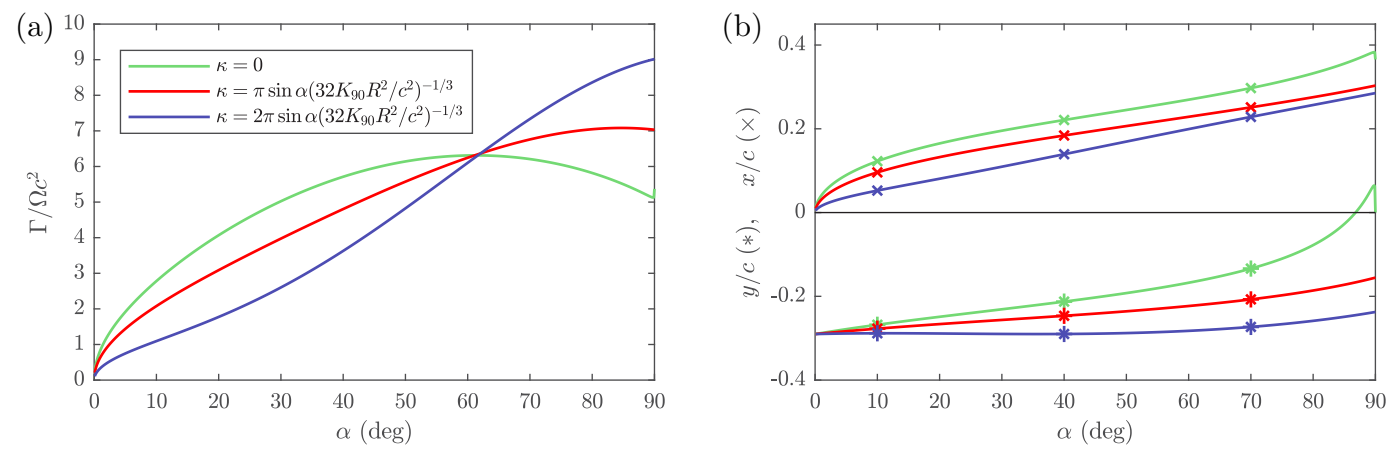

FIG. 6. The effect of the downwash parameter $\kappa$ on the LEV (a) circulation and (b) centroid position, shown for $\operatorname{Re}=500, R / c=4, r / c=2$, and $\alpha$ varied between 0 and $90^{\circ}$.

\section{E. Bio-inspired wing shapes}

Equations (13) and (14) can be used not only for rectangular wings, but also for any wing shape as long as the local chord is wider than the LEV. It was shown [21] that fruit fly, bumblebee, and hawkmoth wing shapes generated nominally the same shape of LEVs. The LEV cone angle mainly depends on Re and $\alpha$. In Fig. 7, panels (a), (b), and (c) correspond to fruit fly, bumblebee, and hawkmoth, respectively. The solid lines with markers show the circulation calculated using available CFD data [21]. Dashed lines show the respective theoretical estimates, which are remarkably close: the r.m.s. difference in $\Gamma$ with respect to the CFD is no greater than $1.9 \Omega c^{2}$.

Note that (14) yields almost identical estimates for the bumblebee and the hawkmoth, with slight discrepancy due to different values of $R / c$ in (17). Both theoretical lines visually coincide with the simplified power-law curve (18). This illustrates the virtue of normalizing $\Gamma$ using $\Omega c^{2}$ and $r$ using
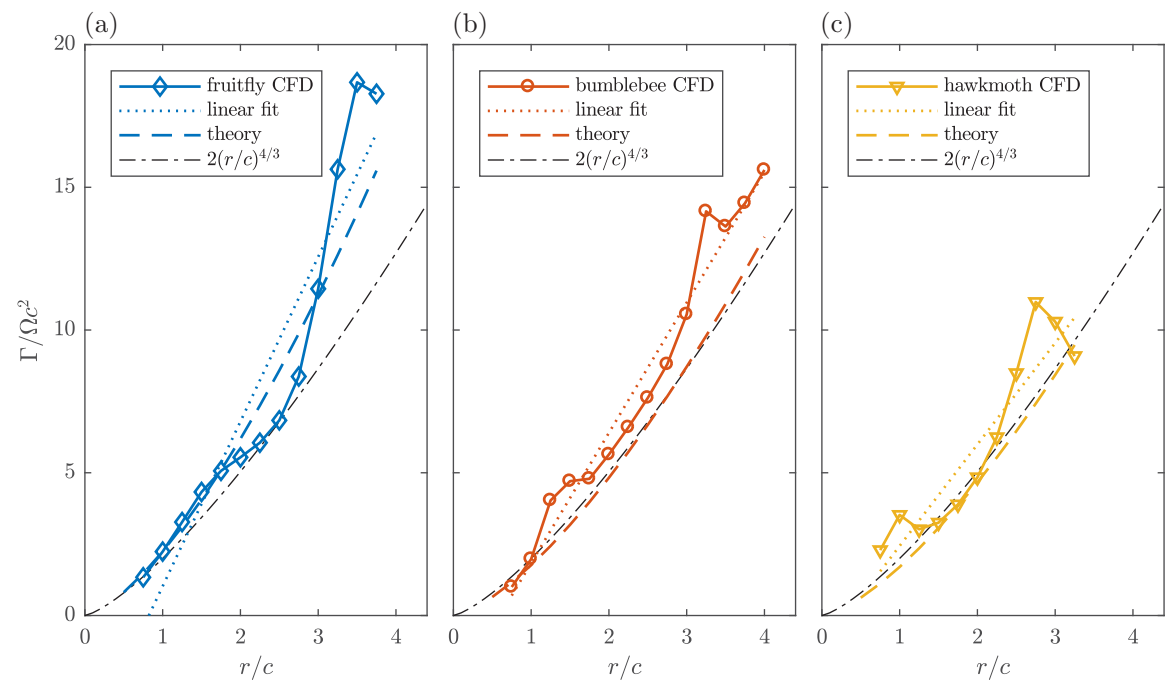

(d)
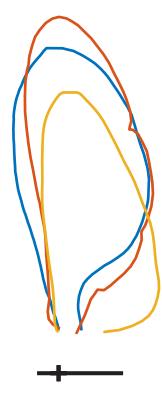

FIG. 7. (a) Fruit fly, (b) bumblebee, and (c) hawkmoth LEVs, at their respective Re equal to 100, 2800, and 5400, at $\alpha=40^{\circ}$. Dimensionless circulation $\Gamma / \Omega c^{2}$ is plotted as a function of dimensionless distance from the rotation axis, $r / c$, where $c$ is the mean chord length for each species. Continuous, dotted, and dashed lines show the CFD data, linear fit to it, and the theoretical estimates, respectively. A reference line $2(r / c)^{4 / 3}$, plotted with dash-dot, is displayed for the ease of comparison between the different cases. (d) The three wing contours, normalized by $c$ (black horizontal scale bar), superposed, and having the same pivot point (black marker). 
$c$, as follows from our analysis. Even though the theory does not account for local features related with variation in $c(r)$, the net growth of $\Gamma$ with $r$ described by (14) is ubiquitous.

For the fruit fly, the theoretical $\Gamma$ grows significantly faster with $r$ because of weaker spanwise vorticity drainage at low Re. This trend may be not immediately visible from the CFD data, but it is apparent from linear regression slope: 3.6 for the hawkmoth, 4.6 for the bumblebee, and 5.8 for the fruit fly.

\section{CONCLUSIONS AND PERSPECTIVES}

Surprising or not, a simple analytical model based only on the vorticity production at the edge and its three-dimensional transport successfully describes the LEV in a wide region of the parameter space. This finding extends on similar results for a rectangular plate perpendicular to the flow [15]. A corollary is that other physical effects such as vortex splitting, breakdown, or secondary vorticity generation at the wing due to the presence of the LEV act to redistribute the vorticity without any significant change of its zeroth and first moments. If the LEV has multiple cores, the line vortex substitutes for all of them combined, i.e., the mentioned effects are taken into account implicitly, and this is sufficient for predicting the circulation. Specialized explicit models may become a necessity when one needs to know how the vorticity is distributed within the LEV.

It should be mentioned that the idea of the vorticity "being created and separated at the leading edge to eventually form a quasi-steady separation bubble ...within which the flow is three-dimensional with a strong flow component along the axis of the vortex that removes vorticity from the neighborhood of the wing" can be traced back to the pioneering work by Maxworthy [3]. Our model is a mathematical formulation of it.

Another important insight gained from the modeling is the use of the dimensionless quantities $\Gamma / \Omega c^{2}, x / c, y / c$, and $r / c$. They primarily depend on $\alpha$ and $\operatorname{Re}_{1}=\Omega c^{2} / \nu$, all other factors being less influential. Moreover, $\mathrm{Re}_{1}$ enters in the equations only through the vorticity transport coefficient $K_{s p}$ and only when $\mathrm{Re}_{1}<105$. In other words, the LEV properties are independent of the Reynolds number as long as it is sufficiently high. This sets an upper bound on the maximum spanwise extent of a stable LEV.

The induced downwash appears to be an important effect when the angle of attack is greater than 0 and less than $90^{\circ}$. With simplifying assumptions on the spanwise distribution of the induced downwash angle, it is possible to parametrize the downwash strength using a single parameter $\kappa$. Increasing $\kappa$ acts to decrease $\Gamma$ for small $\alpha$, but the effect is opposite for large $\alpha$. During the first revolution of the wing, it can be recommended to evaluate $\kappa$ using a simple vortex-ring model (17).

To measure the LEV circulation and track its trajectory using a velocity field obtained in a PIV experiment or a CFD simulation is not a trivial task. Equations (13) and (14) can be used as quick estimates prior to precise measurement. An example MATLAB program is included in part 3 of the Supplemental Material [18]. As a rule of thumb, a less accurate but simpler formula $\Gamma / \Omega c^{2}=$ $2(r / c)^{4 / 3}$ holds for $\alpha \approx 45^{\circ}$ and large Re.

It is customary to refer to the LEV force as the aerodynamic force increment associated with a stable LEV. A precise constructive definition can be deduced from a force decomposition theory that would relate the aerodynamic force and the vorticity field (e.g., Ref. [22]). Thus, calculation of the LEV force may become straightforward when the flow field is fully determined. A reduced-order model, however, provides only some incomplete information about the flow field. In particular, the present paper concerns only the vorticity component in the spanwise direction. However, it is probable that the two-dimensional results such as the Kutta-Joukowski theorem cannot be directly used in this three-dimensional setting, and that the vorticity component in the chordwise direction must be taken into consideration. The question of the LEV force modeling remains open for future research.

\section{ACKNOWLEDGMENTS}

D.K. gratefully acknowledges financial support from the JSPS KAKENHI Grant No. JP18K13693. D.C. was partly supported by China Scholarship Council. H.L. was partly supported 
by the JSPS KAKENHI Grant No. 24120007 for Scientific Research on Innovative Areas. The CFD simulations have been performed using the computer clusters provided by an academic-industrial cooperative research center at Chiba University.

[1] D. D. Chin and D. Lentink, Flapping wing aerodynamics: From insects to vertebrates, J. Exp. Biol. 219, 920 (2016).

[2] H. Liu, C. P. Ellington, K. Kawachi, C. van den Berg, and A. P. Willmott, A computational fluid dynamic study of hawkmoth hovering, J. Exp. Biol. 201, 461 (1998).

[3] T. Maxworthy, Experiments on the Weis-Fogh mechanism of lift generation by insects in hovering flight. Part 1. Dynamics of the 'fling,' J. Fluid Mech. 93, 47 (1979).

[4] H. Liu, D. Kolomenskiy, T. Nakata, and G. Li, Unsteady bio-fluid dynamics in flying and swimming, Acta Mech. Sin. 33, 663 (2017).

[5] D. J. Garmann and M. R. Visbal, Dynamics of revolving wings for various aspect ratios, J. Fluid Mech. 748, 932 (2014).

[6] N. Phillips, K. Knowles, and R. J. Bomphrey, The effect of aspect ratio on the leading-edge vortex over an insect-like flapping wing, Bioinspiration Biomimetics 10, 056020 (2015).

[7] Z. R. Carr, A. C. DeVoria, and M. J. Ringuette, Aspect-ratio effects on rotating wings: Circulation and forces, J. Fluid Mech. 767, 497 (2015).

[8] Y. J. Lee, K. B. Lua, and T. T. Lim, Aspect ratio effects on revolving wings with Rossby number consideration, Bioinspiration Biomimetics 11, 056013 (2016).

[9] D. Tudball Smith, D. Rockwell, J. Sheridan, and M. Thompson, Effect of radius of gyration on a wing rotating at low Reynolds number: A computational study, Phys. Rev. Fluids 2, 064701 (2017).

[10] N. Phillips, K. Knowles, and R. J. Bomphrey, Petiolate wings: Effects on the leading-edge vortex in flapping flight, Interface Focus 7, 20160084 (2017).

[11] T. Jardin and T. Colonius, On the lift-optimal aspect ratio of a revolving wing at low Reynolds number, J. R. Soc. Interface 15, 20170933 (2018).

[12] T. Maxworthy, The formation and maintenance of a leading-edge vortex during the forward motion of an animal wing, J. Fluid Mech. 587, 471 (2007).

[13] E. Limacher, C. Morton, and D. Wood, On the trajectory of leading-edge vortices under the influence of Coriolis acceleration, J. Fluid Mech. 800, R1 (2016).

[14] T. Nakata, H. Liu, and R. J. Bomphrey, A CFD-informed quasi-steady model of flapping-wing aerodynamics, J. Fluid Mech. 783, 323 (2015).

[15] D. Chen, D. Kolomenskiy, and H. Liu, Closed-form solution for the edge vortex of a revolving plate, J. Fluid Mech. 821, 200 (2017).

[16] C. E. Brown and W. H. Michael, Effect of leading-edge separation on the lift of a delta wing, J. Aeronaut. Sci. 21, 690 (1954).

[17] L. Cortelezzi, On the unsteady separated flow past a semi-infinite plate: Exact solution of the Brown and Michael model, scaling, and universality, Phys. Fluids 7, 526 (1995).

[18] See Supplemental Material at http://link.aps.org/supplemental/10.1103/PhysRevFluids.3.114703 for numerical validation of the CFD simulations, description of the vortex identification method in the CFD, and an example computer program for numerical evaluation of the analytical results.

[19] A. Widmann and C. Tropea, Parameters influencing vortex growth and detachment on unsteady aerodynamic profiles, J. Fluid Mech. 773, 432 (2015).

[20] J. W. Kruyt, G. F. van Heijst, D. L. Altshuler, and D. Lentink, Power reduction and the radial limit of stall delay in revolving wings of different aspect ratio, J. R. Soc. Interface 12, 20150051 (2015).

[21] D. Chen, D. Kolomenskiy, T. Nakata, and H. Liu, Forewings match the formation of leading-edge vortices and dominate aerodynamic force production in revolving insect wings, Bioinspiration Biomimetics 13, 016009 (2018).

[22] C.-T. Hsieh, C.-F. Kung, C. C. Chang, and C.-C. Chu, Unsteady aerodynamics of dragonfly using a simple wing-wing model from the perspective of a force decomposition, J. Fluid Mech. 663, 233 (2010). 